Artículo

\title{
Análisis de la eficiencia y retornos de escala de los ingenios azucareros de México
}

\author{
Ramón Valdivia Alcala ${ }^{1}$ \\ Cristóbal Martín Cuevas Alvarado ${ }^{2 \S}$ \\ Juan Salvador Vázquez Muñoz ${ }^{1}$ \\ Mario Ivan García Hernández ${ }^{1}$ \\ Ilich Miguel Santiago Zarate ${ }^{1}$ \\ Ángeles Suhgey Garay Jácome ${ }^{1}$
}

${ }^{1}$ Programa de Postgrado de la División de Ciencias Económico Administrativas-Universidad Autónoma Chapingo. Carretera México-Texcoco km 38.5, Chapingo, Texcoco, Estado de México, México. CP. 56230.

Tel. 595 9517058. (ramvaldi@gmail.com; sazami.09@gmail.com; ivan.garcya@gmail.com; anllely0608@gmail.com). ${ }^{2}$ Consultor-Investigador Independiente. Calle Arboledas 28, Montecillo, Texcoco, Estado de México. CP. 56264. Tel. 5561651251.

${ }^{\S}$ Autor para correspondencia: cuevas_cristobal@hotmail.com.

\section{Resumen}

En México la agroindustria de la caña de azúcar es importante en la economía de 25 entidades federativas y 267 municipios. En la zafra 1995-1996 operaron 61 ingenios azucareros y en la zafra 2017-2018 solo 51 ingenios. En un periodo de 19 años cerró 16.4\% de la infraestructura de molienda de la agroindustria cañera mexicana. Técnicamente existen ineficiencias en sus parámetros que influyeron en su cierre. La presente investigación tiene el objetivo de analizar la eficiencia y naturaleza de los rendimientos a escala con que operaron los ingenios azucareros en México en la zafra 2010-2011. La metodología utilizada fue análisis de datos envolventes (DEA). Los resultados indican que 23 ingenios operaron en su escala óptima, nueve ingenios con eficiencia total, pero con ineficiencia pura e ineficiencia de escala y un tercer grupo que operó con ineficiencia total, ineficiencia pura e ineficiencia de escala. En cuanto a la naturaleza de los rendimientos a escala la DEA permitió identificar que 23 ingenios operaron con rendimientos constantes a escala, 29 con rendimientos crecientes a escala y solo dos ingenios con rendimientos decrecientes a escala. La principal conclusión indica que los 29 ingenios con rendimientos crecientes a escala pueden mejorar su desempeño si ampliaran su tamaño, la producción y la productividad de los factores se incrementaría más que proporcionalmente. El ingenio Pánuco es el único que se identificó tanto con ineficiencia de escala como con rendimientos decrecientes a escala por lo que tal desempeño podría llevarlo a dejar de operar y cerrar como planta.

Palabras clave: análisis de datos envolventes, análisis de frontera estocástica, economías a escala, productividad total de los factores.

Recibido: noviembre de 2021

Aceptado: febrero de 2022 


\section{Introducción}

De acuerdo con el CONADESUCA (2019) la caña de azúcar en México es un cultivo relevante porque la agroindustria de dicho cultivo tiene presencia en 15 entidades federativas y 267 municipios y genera un número importante de empleos directos e indirectos. De acuerdo con la CEMA (2017) en la zafra 2016/2017 en la zafra 2016-2017 participaron 184171 productores de los cuales $71.5 \%$ corresponde a ejidatarios, $24.6 \%$ a pequeños propietarios y $3.9 \%$ a otro tipo de productores como arrendatarios y parcelas escolares. Para la misma zafra CONADESUCA (2019) reporto 68365 cortadores participante. De esta manera, bajo el supuesto de que los productores se emplearon directamente en la zafra y que los cortadores se pueden considerar efectivamente como empleados entonces se estará hablando de 252536 empleos directos.

Además, si se supone también que por cada empleo directo se generan tres empleos directos, entonces se estaría hablando la generación de 1010144 empleos generados en el eslabón de campo de la agroindustria de la caña de azúcar. Ahora bien, en la zafra 2018-2019 se industrializaron 804 060 ha con una producción de azúcar de 6.4 millones de toneladas. De acuerdo con el SIAP (2019) la caña de azúcar representó en el año agrícola 2019 el $6.8 \%$ del valor total de la producción agrícola, ocupando el tercer lugar, tan solo detrás del maíz y aguacate que representaron $17.7 \%$ y $7.7 \%$, respectivamente.

El cultivo de la caña de azúcar es el único cultivo en México que cuenta con una norma que regula las relaciones entre los ingenios cañeros y los agricultores productores de caña de azúcar. Esta norma es la Ley de Desarrollo Sustentable de la Caña de Azúcar publicada en 2005 (SAGARPA, 2005) que junto con el Programa Nacional de la Agroindustria de la Caña (SAGARPA, 2014) son los instrumentos normativos y que dan directrices para la planeación, modernización y mejora de competitividad vía la investigación del cultivo.

No obstante, aunque se cuenta con tal programa específico para la agroindustria en la práctica, la operatividad, rentabilidad y el impulso a la capacidad fabril de la agroindustria presenta desafíos emanados de la casi permanente crisis estructural del sector cañero. En este sentido, ha habido dos momentos en que la situación de los ingenios azucareros, que ha hecho crisis, lo que ha provocado la intervención del sector público. Entre la segunda década del siglo XX y en la de década de los 80s del mismo siglo, los ingenios azucareros fueron administrados por el Estado.

La característica de estos periodos fue la aplicación de subsidios y una política de negocios errática. La situación empeoró entre 1988 y 1990 pues los grandes volúmenes de importaciones de azúcar causaron un exceso de oferta los ingenios estaban sobre endeudados y con cartera vencida, tenían limitado acceso a créditos, altos costos de producción y la fijación de precios era castigada hasta con $15 \%$ por debajo del precio concertado. Aunado a lo anterior existía estancamiento en la productividad de los campos cañeros e ingenios con pérdida de rentabilidad, lo que dificultó el pago de deudas, por lo que varios ingenios cerraron definitivamente.

En este contexto es que en 1988 el gobierno tomó la decisión de vender los ingenios a un grupo de empresarios, entre los que se encontraban socios de las compañías refresqueras, con el argumento de la modernización de la agroindustria (Molina, 2019). 
La segunda intervención del sector público en la agroindustria cañera se suscitó en 2001. De acuerdo con Molina (2019), el gobierno decidió estatizar 27 ingenios con el argumento de paliar la crisis que en ese momento registraban los ingenios, que arrastraban deudas fiscales, de seguridad social, por créditos y con productores y jornaleros, que ascendían a más de tres mil millones de dólares. El corolario de tales crisis ha sido una infraestructura y tecnología productiva poco eficientes, elevados costos de producción y una escasa diversificación en el uso de los coproductos y subproductos obtenidos en el ingenio azucarero y la destilería.

De acuerdo con la Unión Nacional de Cañeros, Asociación Civil, vinculados a la Confederación Nacional de Propietarios Rurales (UNC-CNPR, 1996) la superficie de caña industrializada en la zafra 1995-1996 fue de 577106 ha con un rendimiento de $69.63 \mathrm{t} \mathrm{ha}^{-1}$ y producción de caña molida neta de 39415468 t producida en 61 ingenios. Ahora bien, en la zafra de 1996 operaron 61 ingenios azucareros mientras que en la zafra de 2915 operaron 51. De esta manera en un periodo de 19 años dejaron de operar 10 ingenios en la agroindustria de la caña de azúcar; es decir, un promedio de aproximadamente un ingenio dejo de operar o cerro cada dos años.

El análisis de una serie de indicadores de la productividad de la agroindustria cañera muestra que ésta ha tenido una serie de ineficiencias y atrasos tecnológicos que han repercutido en su rentabilidad y probablemente influir en el cierre de $22 \%$ de los ingenios existentes en un periodo de 19 años. De acuerdo con los Fideicomisos Instituidos en relación con la Agricultura (2016) la caña de azúcar es un insumo que podría generar productos como biofertilizantes, biocombustibles, bonos de carbono por la reducción de emisiones contaminantes, etc.

Esto podría generar ingresos adicionales y mejoraría la posición de rentabilidad de los ingenios. No obstante, la agroindustria esta poco diversificada y además muestra ineficiencias en varios parámetros técnicos. Entre las principales se tiene: Las plantas industriales sólo han aprovechado el 39\% del contenido calórico total de una tonelada de caña (1.2 millones de kilocalorías). La agroindustria de la caña de azúcar compra aproximadamente $6.3 \%$ de energía eléctrica a la Comisión Federal de Electricidad (CFE) para poder realizar la molienda de la caña de azúcar, lo que eleva sus costos de operación. Es decir, la agroindustria cañera no es autosuficiente en la generación de energía eléctrica para autoconsumo, aun cuando el marco legal existente le permite generar excedentes mediante cogeneración y venderlo a la CFE, lo que podría constituir una fuente de ingresos que mejoren sus balances financieros.

El tiempo perdido promedio por zafra en el periodo 2005 a 2016 del total de horas de zafra por año (209 818 horas) es de $19.1 \%$. Este $19.1 \%$ se distribuye como sigue: $3.3 \%$ a campo, $8.9 \%$ a fábrica, $0.4 \%$ por días festivos, $5.4 \%$ por lluvias y $1.1 \%$ por aspectos atribuibles al personal. La mayor parte del tiempo perdido total corresponde al ingenio como tal debido a la escasez de mantenimiento de la planta y de sustitución del equipo problemático u obsoleto. En el periodo 2005 a 2016 la agroindustria de la caña de azúcar ha pagado en promedio $118.28 \mathrm{~kg}$ de azúcar por tonelada de caña molida neta a los productores; no obstante, los kilos de azúcar realmente obtenidos en promedio por tonelada de caña molida neta fueron $117.38 \mathrm{~kg}$.

El ingenio no está alcanzando la eficiencia señalada en la Ley de Desarrollo Sustentable de la caña de azúcar para el pago de la caña en base al precio de referencia determinado por CONADESUCA por lo que el ingenio está pagando un sobre costo de $0.765 \mathrm{~kg} \mathrm{t}^{-1}$ de caña de azúcar que en términos monetarios ha significado una erogación adicional de 3044740 millones de pesos. 
Un parámetro que muestra el atraso tecnológico de los equipos físicos de los ingenios se refleja en el llamado consumo específico de vapor de la caña de azúcar; una utilización totalmente ineficiente del bagazo de caña y del petróleo, por el uso de calderas y turbogeneradores que operan a muy bajas presiones y temperaturas de vapor.

Como mencionó, en el periodo 1995-1996 operaron 61 ingenios azucareros mientras que en la zafra 2015-2016 solo lo hicieron 51, en un periodo de 19 años cerraron 10 ingenios, lo que representa $19.6 \%$ de estas unidades, por lo que resulta de interés el análisis de los 51 ingenios azucareros que operaron en la zafra 2015-2016.

La metodología del análisis envolvente de datos se ha utilizado para medir la eficiencia en el uso de los recursos en diversas actividades económicas. Becerril et al. (2011), la aplicaron para medir la eficiencia técnica del sector agropecuario mexicano. Fontalvo y De la Hoz (2016), la usaron para medir la eficiencia de hospitales en una ciudad colombiana; Quintanilha et al. (2012), para medir la eficiencia de compañías aéreas brasileñas, usaron esta metodología, también se ha usado para medir la eficiencia en cooperativas de crédito (Belmonte y Plaza, 2008) y en la medición de cultivos como el café en Colombia (Perdomo y Mendieta, 2007). Jaforullah y Whiteman (1999), usaron la metodóloga del análisis de datos envolventes para medir la eficiencia de escala de la industria lechera de Nueva Zelanda y examinar la relación entre el tamaño de la granja y la eficiencia técnica.

Tetteh et al. (2016) hacen una evaluación de la eficiencia técnica y la eficiencia de escala de los productores de arroz de pequeña escala en el norte de Ghana así como del efecto del tamaño de la granja sobre la eficiencia, empleando el análisis envolvente de datos para medir la eficiencia. En este contexto, el presente estudio tiene como objetivo evaluar el desempeño de la eficiencia de los ingenios azucareros de México para la zafra 2010-2011 estimando la eficiencia de escala y la naturaleza de los retornos de escala a la que operaron en dicha zafra y determinar cuáles ingenios podrían dejar de operar dadas sus ineficiencias.

\section{Materiales y métodos}

Para la determinación de la eficiencia y de la naturaleza de los rendimientos a escala en la investigación se utilizó el análisis de datos envolventes. El concepto de eficiencia técnica, en el contexto del análisis datos envolventes fue iniciado por Charnes et al. (1978), cuyo desafío fue originalmente el estimar tal concepto de eficiencia técnica considerando múltiples insumos, múltiples productos y sin la usual información de precios. El resultado de aquel trabajo fue la formulación del modelo Charnes-Cooper-Rhodes (CCR) del DEA en forma de un cociente o de programación fraccional.

En contraste con los enfoques paramétricos, cuyo objetivo es optimizar un solo plano de regresión a través de los datos, la DEA optimiza un 'modelo' para cada observación individual con el objetivo de calcular una frontera por partes discreta determinada por el conjunto de las unidades de toma de decisiones (DMU) Pareto-eficientes. Ambos enfoques, el paramétrico como el no paramétrico usan toda la información contenida en los datos. En el análisis paramétrico la línea de regresión optimizada se asume que aplica a cada una de DMU. 
El análisis DEA optimiza la medida, en contraste, del desempeño de cada DMU. Esto resulta en un entendimiento revelado acerca de cada DMU en lugar de la típica DMU promedio. En otras palabras, el foco de la DEA esté en las observaciones individuales; es decir, observación por observación, en contraste con el enfoque de promedios y estimación de parámetros que son asociados con los enfoques estadísticos de una optimización única (Charnes et al., 1994).

El CCR asume, como se ha mencionado, retornos constantes a escala (CRS). Este modelo es apropiado cuando todas las firmas analizadas operan en la escala óptima. Charnes et al. (1978) definen a la eficiencia como el máximo de un cociente de productos ponderados entre insumos ponderados sujeto a que los cocientes similares para cada DMU sean menores o iguales a la unidad. De acuerdo con Huguenin (2012) en forma matemática estas ideas pueden expresarse como sigue: $\mathrm{TE}_{\mathrm{K}}=\frac{\sum_{\mathrm{r}=1}^{\mathrm{s}} \mathrm{u}_{\mathrm{r}} \mathrm{y}_{\mathrm{rk}}}{\sum_{\mathrm{i}=1}^{\mathrm{m}} \mathrm{v}_{\mathrm{i}} \mathrm{x}_{\mathrm{ik}}}$. Donde: $\mathrm{TE}_{\mathrm{k}}$ es la eficiencia tecnica relativa de la firma $\mathrm{k}$ que usa $\mathrm{k}$ insumos para producir $s$ productos $\mathrm{y}$ ademas; $\mathrm{y}_{\mathrm{rk}}=$ cantidad de producto $\mathrm{r}$ producido por la firma $\mathrm{k}$; $\mathrm{x}_{\mathrm{ik}}=$ cantidad de insumo i consumido por la fima $\mathrm{k}$; $\mathrm{u}_{\mathrm{r}}=$ ponderador del producto $\mathrm{r} ; \mathrm{v}_{i}=$ ponderador del insumo $\mathrm{i} ; \mathrm{s}=$ número de productos; $\mathrm{m}=$ número de insumos.

La eficiencia técnica de la empresa $\mathrm{k}$ se maximiza bajo dos restricciones. Primero, las ponderaciones aplicadas a los productos e insumos de la empresa $\mathrm{k}$ no pueden generar un puntaje de eficiencia mayor a 1 cuando se aplica a cada empresa en el conjunto de datos. Segundo, los ponderadores de los productos e insumos son estrictamente positivos. De esta manera para cada firma se debe resolver el siguiente problema de programación lineal: maximizar $\frac{\sum_{\mathrm{r}=1}^{\mathrm{s}} \mathrm{u}_{\mathrm{r}} \mathrm{y}_{\mathrm{rk}}}{\sum_{\mathrm{i}=1}^{\mathrm{m}} \mathrm{v}_{\mathrm{i}} \mathrm{x}_{\mathrm{ik}}}$ sugeto a $\frac{\sum_{r=1}^{s} u_{r} y_{r j}}{\sum_{i=1}^{m} v_{i} x_{i j}} \leq 1 j=1, \ldots, n ; u_{r}, v_{i}>0 \quad \forall r=1, \ldots, s ; i=1, \ldots, m$.

La notación de este problema de programación fraccional es la misma descrita en en los dos últimos párrafos. Éste problema de programación lineal puede ser tratado siguiendo dos enfoques diferentes. En el primer caso, las sumas ponderadas del insumos son minimizadas manteniendo los productos constantes (modelo producto orientado). En el segundo caso, las sumas ponderadas de insumos son maximizadas manteniendo los insumos constantes (modelo insumo orientado).

Como se sabe, la solución se puede obtener al procesar el primal o el dual del problema de programación lineal. Las ecuaciones del primal para cada modelo, expresadas en su forma multiplicativa, son las siguientes y tambien la notación es la descrita para la expresión de la eficiencia tecnica relativa de la unidad $\mathrm{k}\left(\mathrm{TE}_{\mathrm{k}}\right)$.

Modelo CRS orientado al producto

$$
\operatorname{Min} \sum_{\mathrm{i}=1}^{\mathrm{m}} \mathrm{v}_{\mathrm{i}} \mathrm{x}_{\mathrm{ik}} \text { Sujeto a } \sum_{\mathrm{i}}^{\mathrm{m}} \mathrm{v}_{\mathrm{i}} \mathrm{x}_{\mathrm{ij}}-\sum_{\mathrm{r}=1}^{\mathrm{s}} \mathrm{u}_{\mathrm{r}} \mathrm{y}_{\mathrm{rj}} \geq 0
$$

$\mathrm{j}=1, \ldots, \mathrm{n} \sum_{\mathrm{r}=1}^{\mathrm{s}} \mathrm{u}_{\mathrm{r}} \mathrm{y}_{\mathrm{rk}}=1 \mathrm{u}_{\mathrm{r}}, \mathrm{v}_{\mathrm{i}}>0$

$\forall \mathrm{r}=1, . . \mathrm{s} ; \mathrm{i}=1, \ldots, \mathrm{m}$
Modelo CRS orientado al insumo

$$
\begin{aligned}
& \operatorname{Max} \sum_{\mathrm{r}=1}^{\mathrm{s}} \mathrm{u}_{\mathrm{r}} \mathrm{y}_{\mathrm{rk}} \text { Sujeto a } \sum_{\mathrm{i}=1}^{\mathrm{m}} \mathrm{v}_{\mathrm{i}} \mathrm{x}_{\mathrm{ij}}-\sum_{\mathrm{r}=1}^{\mathrm{s}} \mathrm{u}_{\mathrm{r}} \mathrm{y}_{\mathrm{rj}} \geq 0 \\
& \mathrm{j}=1, . ., \mathrm{n} \quad \sum_{\mathrm{i}=1}^{\mathrm{m}} \mathrm{v}_{\mathrm{i}} \mathrm{x}_{\mathrm{ik}}=1 \mathrm{u}_{\mathrm{r}}, \mathrm{v}_{\mathrm{i}}>0 \\
& \forall \mathrm{r}=1, \ldots, \mathrm{s} ; \mathrm{i}=1, \ldots, \mathrm{m}
\end{aligned}
$$

Los materiales de los que se obtuvo la información para alimentar los modelos de programación matemática, en los que está basado el método del análisis de datos envolventes fue el 'informe estadístico del sector agroindustrial de la caña de azúcar en México Zafras 2006-2007-2015-2016’ (CONADESUCA, 2017). Este documento contiene abundante información oficial de la caña de azúcar tanto en campo como en fábrica. 
La información oficial se contrastó con la información publicada por la UNC-CNPR (2017), que es una de las dos organizaciones abastecedoras de materia prima para los ingenios azucareros y representa a los pequeños propietarios que cultivan la caña de azúcar. El periodo seleccionado de la zafra 2010-2011 es porque la información de todos los ingenios está completa y no contiene datos perdidos. Es necesario remarcar que en este estudio se utilizaron datos de corte transversal; es decir, para un solo periodo considerando todas las unidades de toma de decisiones (DMU) o ingenios azucareros.

El software utilizado para correr los modelos del análisis envolvente de datos fue el Data Envelopment Analysis Program 2.1 (DEAP 2.1) de Coelli (1996). Tetteh et al. (2016) se señala que el enfoque DEA genera una superficie envolvente de datos al vincular los puntos en el espacio insumo-producto de tal manera que ya no permite la producción de más producto utilizando el mismo nivel de insumo o la producción del mismo producto utilizando menos insumos. La superficie de envoltura de datos sirve como punto de referencia para medir la eficiencia relativa del resto de empresas debajo de la superficie de envoltura.

Empíricamente esto significa que todas las unidades eficientes están unidas por un continuo para formar una frontera eficiente en la cual el puntaje de eficiencia para cada unidad de toma de decisiones se mide por lo lejos que se desvía de la frontera eficiente.

La frontera en el caso de los rendimientos constantes a escala es lineal, mientras que para los rendimientos variables a escala es una envoltura por piezas convexa. Es posible descomponer el análisis de eficiencia en tres dimensiones para comprender las posibles fuentes de ineficiencia. La primera dimensión se relaciona con la eficiencia técnica global, la segunda se relaciona con la eficiencia técnica pura, mientras que la tercera se relaciona con la eficiencia de escala.

La estimación de la eficiencia técnica global implica medir la relación entre la distancia entre los puntos ineficientes y la frontera eficiente de retornos constantes a escala (CRS), mientras que la estimación de la eficiencia técnica pura implica medir la relación entre la distancia entre puntos ineficientes y la frontera eficiente bajo rendimientos variables a escala (VRS). La eficiencia de escala (SE) es la relación entre la eficiencia técnica global (OTE) y la eficiencia técnica pura (PTE). El estudio de Tetteh et al. (2016) emplea la orientación al insumo en la estimación porque los productores tienen más control sobre los insumos que sobre la producción.

\section{Resultados y discusión}

De acuerdo con el CONADESUCA (2018) en la zafra 2015-2016 la agroindustria de la caña de azúcar en México operó con 51 ingenios cañeros. De éstos, en Veracruz se localizan 18 (35.3\%), en Jalisco 6 (11.8\%), en San Luis Potosí 4 (7.8\%), en Michoacán 3 (5.9\%) y en Oaxaca 3 (5.9\%). Estas cinco entidades agrupan al $66.7 \%$ de la agroindustria de la caña de azúcar en México.

Las variables utilizadas para construir el modelo DEA basado en la programación lineal, calcular la eficiencia técnica de las unidades de toma de decisiones bajo retornos constantes a escala, retornos variables a escala y la eficiencia de escala se presentan en el Cuadro 1, mientras que los estadísticos descriptivos de tales variables se muestran en el (Cuadro 2). Las unidades de las variables son las mostradas en la segunda columna del (Cuadro 1). 
Cuadro 1. Definición de las variables utilizadas.

\begin{tabular}{ccc}
\hline Variable & Unidades & Tipo \\
\hline Azúcar producida por hectárea & Toneladas & Output \\
Cachaza & Toneladas & Output \\
Energía total consumida & $\mathrm{Kw}-\mathrm{h}$ & Input \\
Vehículos de acarreo & Unidades & Input \\
Caña molida neta & Toneladas & Input \\
Cortadores & Jornaleros & Input \\
Frentes de corte & Unidades & Input \\
Tiempo de zafra perdido & Horas & Input \\
\hline
\end{tabular}

CONADESUCA (2017).

Cuadro 2 Estadísticas descriptivas de las variables utilizadas zafra 2010-2011.

\begin{tabular}{ccccc}
\hline Variable & Promedio & Valor máximo & Valor mínimo & $\begin{array}{c}\text { Coeficiente de } \\
\text { variación }(\%)\end{array}$ \\
\hline Azúcar producida por hectárea & 97465 & 228998 & 10482 & 57.5 \\
Cachaza & 112029 & 290372 & 15458 & 68.6 \\
Energía total consumida & 496066300 & 1181558548 & 87691047 & 54.3 \\
Vehículos de acarreo & 298 & 1393 & 31 & 75.2 \\
Caña molida neta & 12628 & 35865 & 1523 & 58.3 \\
Cortadores & 796046 & 1760707 & 122682 & 54.1 \\
Frentes de corte & 1320 & 3839 & 76 & 64.4 \\
Tiempo de zafra perdido & 3427 & 5616 & 1087 & 25.3 \\
Azúcar producida por hectárea & 517 & 1361 & 67 & 52 \\
\hline
\end{tabular}

CONADESUCA (2017).

Una vez que se realizó un análisis exploratorio de los datos y analizando los estadísticos descriptivos para detectar observaciones que podrían distorsionar el análisis, se procesó el modelo DEA para el cálculo de la eficiencia técnica bajo el supuesto de retornos constantes a escala y el modelo DEA bajo el supuesto de retornos variables a escala. Como se mencionó arriba, la eficiencia de escala se obtiene como el cociente de la eficiencia técnica (bajo el supuesto de retornos constantes a escala) sobre la eficiencia técnica (bajo el supuesto de retornos variables a escala). Antes de proceder con el análisis es necesario mencionar que a la eficiencia obtenida bajo rendimientos constantes a escala se le llama eficiencia total, a la eficiencia obtenida bajo rendimientos variables a escala se le denomina eficiencia pura (Coelli et al., 1998; Coelli y Prasada et al., 2005; Zhu, 2013).

\section{Ingenios operando en escala óptima}

En el grupo de ingenios azucareros que operaron en su escala óptima, es decir aquellos cuyos índices de eficiencia bajo retornos constantes a escala (eficiencia total), retornos variables a escala (eficiencia pura) y la eficiencia de escala son iguales a la unidad, se encontraron a 23 ingenios: 
Adolfo López Mateos, Alianza Popular, Atencingo, Central Casasano, Central Motzorongo, El Higo, El Molino, El Potrero, El Dorado, Emiliano Zapata, La Gloria, Los Mochis, Melchor Ocampo, Nuevo San Francisco, La Margarita, Plan de San Luis, Puga, Pujiltic, San Cristóbal, San Miguel del Naranjo, Tala, Tamazula y Tres Valles. El que un ingenio opere en su escala óptima significa que, los insumos utilizados en el proceso productivo como tierra, unidades de acarreo, cortadores, horas de zafra, energía utilizada para generar potencia de la molienda, etc., están siendo manejados y administrados con las mejores prácticas productivas. La escala óptima implica que los ingenios azucareros están trabajando en su tamaño óptimo.

\section{Ingenios operando con ineficiencia total e ineficiencia de escala}

El segundo grupo de ingenios se encuentran aquellas unidades de toma de decisiones que operaron con una eficiencia total y una eficiencia de escala menor que la unidad. Este grupo tiene ocho ingenios azucareros como se muestra en el (Cuadro 3).

Cuadro 3. Ingenios azucareros operando con ineficiencia total y de escala 2010-2011.

\begin{tabular}{lcccc}
\hline \multicolumn{3}{c}{ Ingenio } & \multicolumn{3}{c}{ Eficiencia } \\
\hline 1 & Azsuremex & 0.6482 & 1 & 0.6482 \\
2 & Calipam & 0.8178 & 1 & 0.8178 \\
3 & Santa Clara & 0.8384 & 1 & 0.8384 \\
4 & Bellavista & 0.8531 & 1 & 0.8531 \\
5 & Pedernales & 0.9129 & 1 & 0.9129 \\
6 & Lázaro Cárdenas & 0.9336 & 1 & 0.9336 \\
7 & José María Morelos & 0.9383 & 1 & 0.9383 \\
8 & San Francisco Ameca & 0.9523 & 1 & 0.9523 \\
\hline
\end{tabular}

En este segundo grupo de ingenios, los indicadores de eficiencia muestran lo siguiente: la eficiencia pura indica que los recursos e insumos utilizados son manejados y administrados con las mejores prácticas productivas. La fuente de ineficiencia de estos ingenios es su tamaño. Por lo tanto, si dichos ingenios ampliaran su escala mejorarían su eficiencia y productividad. Destaca en este caso el ingenio Azsuremex, cuya eficiencia total y eficiencia de escala es baja (64.82\%), por lo que ampliando su escala de operación mejoraría su eficiencia hasta casi $35 \%$.

\section{Ingenios operando con ineficiencia total, ineficiencia pura e ineficiencia de escala}

En un tercer grupo de ingenios se identifican aquellas unidades de toma de decisiones cuyas ineficiencias provienen tanto de un mal manejo de los recursos e insumos que intervienen en el eslabón de campo como del proceso de molienda en el eslabón de fábrica, así como de la ineficiencia de escala; es decir, a causa del tamaño del ingenio. En este grupo se encontraron 22 ingenios, según se muestra en el (Cuadro 4).

En este grupo de ingenios destaca el caso de Santa Rosalía. La ineficiencia mayor de este ingenio se explica en la zafra de estudio por el mal manejo y administración de los recursos productivos y del ingenio, su ineficiencia pura fue de $16 \%$ mientras que su ineficiencia de escala fue $6 \%$. La ineficiencia total de este ingenio fue de $21.32 \%$. Una situación muy parecida tuvo en aquella zafra los ingenios Presidente Benito Juárez, Cuatotolapam, entre otros. 
Cuadro 4. Ingenios azucareros con ineficiencia pura y de escala 2010-2011.

\begin{tabular}{lcccc}
\hline & Ingenio & \multicolumn{3}{c}{ Eficiencia } \\
\hline 1 & El Carmen & 0.8185 & 0.9062 & 0.9032 \\
2 & San Miguelito & 0.8836 & 0.9684 & 0.9124 \\
3 & San Pedro & 0.8436 & 0.9152 & 0.9218 \\
4 & Cuatotolapam & 0.8211 & 0.8792 & 0.9339 \\
5 & Santa Rosalía & 0.7868 & 0.8367 & 0.9404 \\
6 & El Refugio & 0.9409 & 0.9989 & 0.9419 \\
7 & San José de Abajo & 0.8849 & 0.9379 & 0.9434 \\
8 & Mahuixtlán & 0.9033 & 0.9558 & 0.9451 \\
9 & Huixtla & 0.8319 & 0.8799 & 0.9455 \\
10 & El Modelo & 0.8971 & 0.9401 & 0.9543 \\
11 & La Joya & 0.8975 & 0.9386 & 0.9562 \\
12 & Presidente Benito Juárez & 0.8246 & 0.8486 & 0.9717 \\
13 & Central La Providencia & 0.917 & 0.9433 & 0.9721 \\
14 & San Rafael de Pucté & 0.9005 & 0.9117 & 0.9877 \\
15 & Constancia & 0.9082 & 0.9194 & 0.9878 \\
16 & Central Progreso & 0.935 & 0.9449 & 0.9895 \\
17 & El Mante & 0.9328 & 0.9412 & 0.9911 \\
18 & San Nicolás & 0.8943 & 0.9008 & 0.9929 \\
19 & Plan de Ayala & 0.9735 & 0.9784 & 0.995 \\
20 & Aarón Sáenz Garza & 0.9667 & 0.969 & 0.9976 \\
21 & Quesería & 0.9208 & 0.9216 & 0.9991 \\
22 & Pánuco & 0.9675 & 0.968 & 0.9995 \\
\hline
\end{tabular}

\section{Identificación de los rendimientos a escala}

En la identificación de la naturaleza de los rendimientos a escala que tiene cada planta de la agroindustria azucarera nacional juega un papel relevante los llamados retornos a escala no crecientes (NIRS). Este concepto es por sí mismo motivo de estudios completos. No obstante, dado que el software lo calcula automáticamente, es común que se utilice conjuntamente con la eficiencia pura (o bajo retornos variables a escala) y la eficiencia total (o bajo retornos constantes a escala) para identificar qué tipo de rendimientos a escala tienen en este caso, los ingenios mexicanos. A partir de tres reglas es posible identificar la naturaleza de los rendimientos a escala.

Cuando la eficiencia pura es igual a la eficiencia bajo NIRS el ingenio opera bajo rendimientos decrecientes a escala. Cuando la eficiencia pura es igual a la eficiencia total y estas son igual a la eficiencia bajo NIRS el ingenio está operando bajo rendimientos constantes a escala. Cuando la eficiencia total es igual a la eficiencia bajo NIRS el ingenio está operando bajo rendimientos crecientes a escala. En el primer caso se ubicó solo un ingenio azucarero y correspondió al ingenio Pánuco y se muestra en el (Cuadro 5). 
Cuadro 5. Ingenios cañeros con rendimientos decrecientes a escala 2010-2011.

\begin{tabular}{cccccc}
\hline \multirow{2}{*}{ No. Ingenio } & \multicolumn{3}{c}{ Eficiencia } & \multirow{2}{*}{ Naturaleza de los rendimientos a escala } \\
\cline { 3 - 4 } & & Bajo CRS & Bajo VRS & Bajo NIRS & \\
\hline & & (total) & (pura) & & Decrecientes \\
\hline
\end{tabular}

El caso del ingenio Pánuco es el más crítico, pues los rendimientos decrecientes a escala se caracterizan porque si se aumentara su escala (tamaño), la producción aumentará en una menor proporción y además la productividad de los factores disminuirá. Si bien este resultado fue para la zafra 2010-2011, se esperaría que, de haber tenido un comportamiento similar en zafras posteriores, Él mismo podría dejar de operar. El segundo caso corresponde al grupo de grupo de 23 ingenios que operaron en la escala óptima; es decir, la eficiencia total, la eficiencia pura y por lo tanto la eficiencia de escala son iguales a la unidad. Estos ingenios operaron con rendimientos constantes a escala y los mismos mencionados en el primer grupo de la sección anterior.

En el tercer caso se tiene aquellos ingenios que operaron bajo rendimientos crecientes a escala. En este grupo se identificaron 29 ingenios y son los que se muestran en el Cuadro 6. De acuerdo con la teoría económica, los ingenios que presentan rendimientos crecientes a escala pueden mejorar su eficiencia, pues si aumentaran su capacidad instalada, la producción aumentaría en una mayor proporción y la productividad de los factores se incrementaría.

Cuadro 6. Ingenios cañeros con rendimientos crecientes a escala 2010-2011.

\begin{tabular}{cccccc}
\hline \multirow{2}{*}{ Núm. } & Ingenio & \multicolumn{3}{c}{ Eficiencia } & Naturaleza de los \\
\cline { 3 - 4 } & & Bajo CRS & Bajo VRS & Bajo NIRS & rendimientos a escala \\
\hline 1 & Azsuremex & 0.6482 & 1 & 0.6482 & Crecientes \\
2 & Santa Rosalía & 0.7868 & 0.8367 & 0.7868 & Crecientes \\
3 & Calipam & 0.8178 & 1 & 0.8178 & Crecientes \\
4 & El Carmen & 0.8185 & 0.9062 & 0.8185 & Crecientes \\
5 & Cuatotolapam & 0.8211 & 0.8792 & 0.8211 & Crecientes \\
6 & Presidente Benito Juárez & 0.8246 & 0.8486 & 0.8246 & Crecientes \\
7 & Huixtla & 0.8319 & 0.8799 & 0.8319 & Crecientes \\
8 & Santa Clara & 0.8384 & 1 & 0.8384 & Crecientes \\
9 & San Pedro & 0.8436 & 0.9152 & 0.8436 & Crecientes \\
10 & Bellavista & 0.8531 & 1 & 0.8531 & Crecientes \\
11 & San Miguelito & 0.8836 & 0.9684 & 0.8836 & Crecientes \\
12 & San José de Abajo & 0.8849 & 0.9379 & 0.8849 & Crecientes \\
13 & San Nicolás & 0.8943 & 0.9008 & 0.8943 & Crecientes \\
14 & El Modelo & 0.8971 & 0.9401 & 0.8971 & Crecientes \\
15 & La Joya & 0.8975 & 0.9386 & 0.8975 & Crecientes \\
16 & San Rafael de Pucté & 0.9005 & 0.9117 & 0.9005 & Crecientes \\
\hline
\end{tabular}




\begin{tabular}{lccccc}
\hline \multirow{2}{*}{ Núm. } & Ingenio & \multicolumn{3}{c}{ Eficiencia } & Naturaleza de los \\
\cline { 3 - 5 } & & Bajo CRS & Bajo VRS & Bajo NIRS & rendimientos a escala \\
\hline 17 & Mahuixtlán & 0.9033 & 0.9558 & 0.9033 & Crecientes \\
18 & Constancia & 0.9082 & 0.9194 & 0.9082 & Crecientes \\
19 & Pedernales & 0.9129 & 1 & 0.9129 & Crecientes \\
20 & Central La Providencia & 0.917 & 0.9433 & 0.917 & Crecientes \\
21 & Quesería & 0.9208 & 0.9216 & 0.9208 & Crecientes \\
22 & El Mante & 0.9328 & 0.9412 & 0.9328 & Crecientes \\
23 & Lázaro Cárdenas & 0.9336 & 1 & 0.9336 & Crecientes \\
24 & Central Progreso & 0.935 & 0.9449 & 0.935 & Crecientes \\
25 & José María Morelos & 0.9383 & 1 & 0.9383 & Crecientes \\
26 & El Refugio & 0.9409 & 0.9989 & 0.9409 & Crecientes \\
27 & San Francisco Ameca & 0.9523 & 1 & 0.9523 & Crecientes \\
28 & Aarón Sáenz Garza & 0.9667 & 0.969 & 0.9667 & Crecientes \\
29 & Plan de Ayala & 0.9735 & 0.9784 & 0.9735 & Crecientes \\
\hline
\end{tabular}

Al comparar estos resultados con los de otras investigaciones se encontraron dos situaciones. Campos y Oviedo (2015); Pérez (2007); Sen (1962), si bien estudian el tamaño de los predios sobre la productividad de la caña de azúcar por hectárea solo lo hacen mediante estadísticos descriptivos, principalmente la media, lo que provoca que sus resultados cuantitativos no sean comparables con los obtenidos con la metodología del análisis de datos envolventes (DEA).

Otros resultados, aunque se derivan de la metodología DEA para estudiar la eficiencia de escala y la naturaleza de los rendimientos a escala, lo hacen para productos distintos a la caña de azúcar. Por lo que la discusión se reduce solo a contrastar los resultados obtenidos con estudios que analizan otras agroindustrias o sector de la economía. De esta manera se puede afirmar que la presente investigación es pionera en México, en determinar la eficiencia de escala y de rendimientos a escala utilizando la metodología DEA. De esta forma, por ejemplo, Jaforullab y Whiteman (1999) empíricamente descompusieron la eficiencia total en eficiencia pura y de escala en la agroindustria lechera de Nueva Zelanda. Los resultados mostraron que $19 \%$ de las granjas están operando en su escala óptima, 28\% están arriba de su escala óptima y 57\% estaban por abajo de su escala óptima. Un resultado relevante también es que el tamaño de las explotaciones lecheras con el paso del tiempo, aumentan su tamaño, pero decrece su número.

\section{Conclusiones}

Se encontró que 23 ingenios operaron en su escala óptima y también presentaron rendimientos constantes a escala lo que implica que la gestión de los recursos que poseen, son administrados con eficiencia. Se encontró un segundo grupo de ingenios que presentaron rendimientos crecientes a escala, lo que implica que pueden mejorar su eficiencia pues si incrementan la utilización de insumos en una determinada proporción, el producto obtenido se incrementará más que proporcionalmente. En este grupo se encontraron 29 ingenios azucareros. Finalmente, un tercer 
grupo que presento rendimientos decrecientes a escala; es decir, que, si incrementaran los recursos como superficie cosechada, maquinaria, entre otros, su producción de caña disminuirá. En este caso se encuentra el ingenio Pánuco. La conclusión general del estudio es la presencia de mayor cantidad de ingenios con rendimientos crecientes a escala (29) implica que existe oportunidad de mejorar la eficiencia de la agroindustria nacional de la caña de azúcar. El presente estudio es el primero en determinar la eficiencia de escala y la naturaleza de los rendimientos a escala utilizando la metodología de la frontera eficiente en la agroindustria de caña de azúcar en México.

\section{Literatura citada}

Becerril, T. O. U.; Rodríguez, L. G y Ramírez, H. J. J. 2011. Eficiencia técnica del sector agropecuario de México: una perspectiva de análisis envolvente de datos. Economía. 34(31):85-110. https://www.redalyc.org/articulo.oa?id=195621325004.

Belmonte, U. L. J. y Plaza, Ú. J. A. 2008. Análisis de la eficiencia en las cooperativas de crédito en España. Una propuesta metodológica basada en el análisis envolvente de datos (DEA). Rev. Econ. Púb. Soc. Coop. 22(63):113-133. https://ojs.uv.es/index.php/ciriecespana/ index.

CEMA. 2017. Compañía Editora del Manual Azucarero Mexicano. Manual azucarero mexicano. (Ed.) Cía. Editora del Manual Azucarero, SA de CV Ciudad de México, México. 487 p.

Campos, O. F. y Oviedo, P. M. 2015. Extensión de los predios agrícolas y productividad. El caso del campo cañero en México. El Trimestre Económico. 80(325):147-181.

Charnes, A.; Cooper, W. W.; Lewin, A. Y. and Seiford, L. M. 1994. Data envelopment analysis. Theory, methodology, and application. Springer Science+Business Media LLC. New York, NY. 507 p.

Charnes, A.; Cooper, W. W. and Rhodes, E. 1978. Measuring the efficiency of decision-making units. Eur. J. Oper. Res. 2(6):429-444. Doi:https://doi.org/10.1016/0377-2217(78)90138-8.

Coelli, T. 1996. A guide to DEAP. Version 2.1: a data envelopment analysis (computer) program. Centre for Efficiency and Productivity Analysis. University of New England, Australia. http://www.owlnet.rice.edu/ econ380/DEAP.PDF.

Coelli, T. J.; Prasada, R, D. S. P.; O’Donnell, C. J. and Battese, G. E. 2005. An introduction to efficiency and productivity analysis. $2^{\text {th }}$ (Ed.). Springer: New York, NY, USA. 341 p.

Coelli, T. J.; Prasada, R. D. S. and Battese, G. E. 1998. An Introduction to efficiency and productivity analysis. Kluwer Academic Publisher. Boston, Massachusetts, USA. 341 p.

CONADESUCA. 2017. Comité Nacional para el Desarrollo sustentable de la caña de azúcar. Informe estadístico del sector agroindustrial de la caña de azúcar en México Zafras 20062007/2015-2016. Ciudad de México, México. https://www.gob.mx/conadesuca/articulos/ informe-estadistico-del-sector-agroindustrial-de-la-cana-de-azucar-en-mexico-zafras2006-2007-2015-2016?idiom=es.

CONADESUCA. 2018. Comité Nacional para el Desarrollo Sustentable de la Caña de Azúcar. Quinto informe estadístico del sector agroindustrial de la caña de azúcar en México Zafras 2008-2009/2017-2018. Ciudad de México, México. https://www.gob.mx/cms/uploads/ attachment/file/392732/Nacional_y_Entidad_Federativa.pdf.

CONADESUCA. 2019. Comité Nacional para el Desarrollo Sustentable de la Caña de Azúcar. $6^{\circ}$ Informe estadístico del sector agroindustrial de la caña de azúcar en México. Zafras 20092010/2018-2019. Ciudad de México, México. https:/www.gob.mx/conadesuca/es/ articulos/6-informe-estadistico-del-sector-agroindustrial-de-la-cana-de-azucar-en-mexico ?idiom=es. Zafras 2009-2010 / 2018-2019. 
FIRA. 2016. Fideicomisos Instituidos en Relación a la Agricultura. Panorama agroalimentario. Azúcar 2015. Morelia, Michoacán, México. https://www.gob.mx/cms/uploads/attachment/ file/61947/Panorama_Agroalimentario_Az_car_2015.pdf.

Fontalvo, H. T. J. y De la Hoz, H. G. 2016. Eficiencia de los hospitales de Bolívar, Colombia, por medio análisis envolvente de datos. Dimensión Empresarial. 14(1):95-108.

Huguenin, J. M. 2012. Data envelopment analysis (DEA). A pedagogical guide for decision makers in the public sector. Institut de Hautes Études en Administration Publique (IHEAP). Laussane, Swiis. https://serval.unil.ch/en/notice/serval:BIB_0FC432348A97.

Jaforullah, M. and Whiteman, J. 1999. Scale efficiency in the New Zealand dairy industry: a nonparametric approach. The Australian J. Agric. Res. Econ. 43(4):523-541. Doi:10.1111/ 1467-8489.00093.

Molina, E. I. 2019. Iniciativa que reforma diversas disposiciones de la ley de desarrollo sustentable de la caña de azúcar. Sistema de Información Legislativa. Secretaría de Gobernación. http://si1.gobernacion.gob.mx/portal.

Perdomo, J. A. y Mendieta, J. C. 2007. Factores que afectan la eficiencia técnica y asignativa en el sector cafetero colombiano: una aplicación con análisis envolvente de datos. Desarrollo y Sociedad. 2(60):1-46.

Pérez, Z. A. 2007. Tenencia de la tierra e industria azucarera. (Ed.) Porrúa. México, DF. 226 p.

Quintanilha, da S. J.; Baptista, J. C. C. y Angulo, M. L. 2012. Evaluación de la eficiencia de las compañías aéreas brasileñas a través de un modelo híbrido de análisis envolvente de datos (DEA) y programación lineal multiobjetivo. Ingeniare. Rev. Chilena de Ingeniería. 20(3):331-342.

SAGARPA. 2005. Secretaría de Agricultura, Ganadería, Desarrollo Rural Pesca y alimentación. Ley de desarrollo sustentable de la caña de azúcar. Diario Oficial de la Federación (DOF) del 22 de agosto de 2005. DF, México.

SAGARPA-CONADESUCA. 2014. Secretaría de Agricultura, Ganadería, Desarrollo Rural, Pesca y Alimentación-Comité Nacional para el Desarrollo Sustentable de la Caña de Azúcar. Programa Nacional de la Agroindustria de la Caña de Azúcar (PRONAC). Diario Oficial de la Federación (DOF) del 2 de mayo de 2014. DF, México.

Sen, A. K. 1962. An aspect of Indian agriculture. Economic \& Political Weekly. 14(4-6):243-245.

SIAP. 2019. Servicio de Información Agroindustrial y Pesquera. Anuario estadístico de la producción agrícola. Producción agrícola. Año 2019. Ciudad de México, México. https://nube.siap.gob.mx/cierreagricola/.

Tetteh, A. B.; Backman, S. and Rezitis, A. 2016. Does farm size matter? Investigating scale efficiency of peasant rice farmers in northern Ghana. Economics Bulletin. 36(4):22752290.

UNC-CNPR. 2017. Unión Nacional de Cañeros-Confederación Nacional de Propietarios Rurales. Estadisticas de la Agroindustria de Caña de Azúcar. 2005-2006/2015-2016. DF, Méxo.

Zhu, J. 2013. Quantitative models for performance evaluation and benchmarking. Data envelopment analysis with spreadsheets. Springer. New York. 420 p. 